\title{
Genetics of water use physiology in locally adapted Arabidopsis thaliana
}

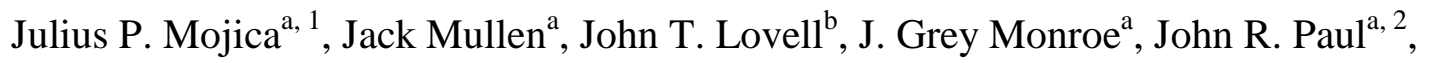 \\ Christopher G. Oakley ${ }^{\mathrm{c}}$, John K. McKay ${ }^{\mathrm{a}}$ \\ ${ }^{\mathrm{a} D e p a r t m e n t ~ o f ~ B i o a g r i c u l t u r a l ~ S c i e n c e s ~ \& ~ P e s t ~ M a n a g e m e n t, ~ C o l o r a d o ~ S t a t e ~ U n i v e r s i t y, ~}$ \\ Fort Collins, CO 80523 USA \\ ${ }^{\mathrm{b}}$ Department of Integrative Biology, University of Texas, Austin, TX 78701, USA \\ ${ }^{\mathrm{c}}$ Department of Plant Biology, Michigan State University, East Lansing, MI 48824, USA \\ ${ }^{1}$ Present address, Department of Biology, Duke University, Durham, NC 27708 USA \\ ${ }^{2}$ Present address, Department of Biology, University of San Francisco, San Francisco, \\ CA 94117, USA
}

Corresponding author: julius.mojica@duke.edu 


\section{Abstract}

Identifying the genetic basis of adaptation to climate has long been a goal in evolutionary biology and has applications in agriculture. Adaptation to drought represents one important aspect of local adaptation, and drought is the major factor limiting agricultural yield. We examined local adaptation between Sweden and Italy Arabidopsis thaliana ecotypes, which show contrasting levels of water availability in their local environments. To identify quantitative trait loci (QTL) controlling water use physiology traits and adaptive trait QTL (genomic regions where trait QTL and fitness QTL colocalize), we performed QTL mapping on $374 \mathrm{~F}_{9}$ recombinant inbred lines in wellwatered and terminal drought conditions. We found 72 QTL (32 in well-watered, 31 in drought, 9 for plasticity) across five water use physiology traits: $\delta^{13} \mathrm{C}$, rosette area, dry rosette weight, leaf water content and \% leaf nitrogen. Some of these genomic regions colocalize with fitness QTL and with each other in defined hotspots. In addition, we found evidence of both constitutive and inducible water use physiology QTL. Finally, we identified highly divergent candidate genes, in silico. Our results suggest that many genes with minor effects may influence adaptation through water use physiology and that pleiotropic water use physiology QTL may have fitness consequences.

Keywords: water-stress, QTL, plasticity, constitutive, inducible, adaptation, fitness

\section{Introduction}

Drought stress is the major limiting abiotic factor to yield in agricultural systems and a major selective force in nature $[1,2]$. Because of this, there is a need to understand the physiological mechanisms and causal genetic polymorphisms that cause drought adaptation (reviewed by Kooyers 2015, [3]).

Plants have evolved diverse physiological mechanisms to survive and reproduce despite soil or atmospheric water deficits. Constitutive water use physiology (e.g. extreme slow growth), or rapid cycling may impart adaption to drought in nature. However, these strategies often antagonize yield when moisture is plentiful [4]. As such, inducible physiological responses to soil moisture, which permit plants to maintain homeostasis despite drought stress, $[5,6]$ may be a more promising target for plant breeding. Such traits, including increased photosynthetic water-use-efficiency (WUE) and root elongation, but reduced leaf growth constitutes a "dehydration avoidance" drought adaptive strategy [7]. By optimizing constitutive dehydration avoidant phenotypes and increasing physiological plasticity of these traits, breeders seek to 
increase survival and yield during drought without incurring a yield cost when moisture is more plentiful (e.g. [8]).

Identifying the genetic basis of dehydration avoidance among crop cultivars has been limited, until recently, by a lack of genomic information and tools for functional genetic manipulation. In contrast, there has been considerable work on the molecular basis of dehydration avoidance in Arabidopsis thaliana [9-16]. However these efforts have largely focused on a single accession (Col-0), which lacks the ecological and evolutionary context to study drought adaptation.

Natural systems offer an opportunity to understand how natural selection has solved the problem of adapting to drought stress. In particular, genetic mapping of physiological traits among populations that are known to be locally adapted has yielded many candidate loci for stress adaptation [17-19]. A particularly fertile example comes from a cross between locally adapted Arabidopsis thaliana (hereforth, A. thaliana) populations from Sweden and Italy [20], where multi-year experiments in the native habitats have identified Quantitative Trait Loci (QTL) underlying lifetime fitness (survival and reproduction). Controlled growth chamber experiments using the same mapping population have further identified loci causing variation in freezing tolerance [21] and flowering time [22], several of which colocalize with QTL involved in local adaptation. Candidate genes underlying these "adaptive trait QTL" (i.e., underlying both fitness and physiological variation) are especially promising targets for efforts to breed and engineer stress tolerant cultivars.

Here we exploit the Sweden-Italy mapping population of $A$. thaliana to examine the genetic basis of drought-inducible and constitutive dehydration avoidance phenotypes, and to identify which of these QTL colocalize with fitness QTL. We measured water use physiology phenotypes (broad set of robust indicators of plant response to soil moisture availability) like carbon isotope ratio, rosette leaf area, dry mass of the rosette, leaf water content and percent leaf nitrogen. We include leaf nitrogen as it is part of the larger question on the evolution of leaf traits and physiology [23] and it has been shown that drought treatment leads to increase in leaf nitrogen in a diverse collection of $A$. thaliana [24]. In a multi-taxa meta analysis, leaf nitrogen showed the strongest trait loading among the multivariate leaf traits (e.g., leaf mass, leaf lifespan, 
photosynthetic capacity, dark respiration rate etc.). Understanding leaf nitrogen's relationship with other physiological traits allows us to elucidate the leaf economics spectrum (sensu Wright 2004, [25]), which will inform breeding for the next generation of drought-adapted crops with efficient leaf investment strategies. We employ a QTL mapping approach in this bi-parental cross and compare the genomes of the two parents in order to identify candidate genes that may underlie differences in water use physiology. Theory predicts that the magnitude of local adaptation is directly proportional to the degree of environmental divergence between two populations [26]. Because the habitats of the Sweden and Italy populations significantly vary in water availability across the major life stages in A. thaliana development, we hypothesize that traits related to water use physiology are important determinants of fitness variation in the field. Specifically, we address three research questions: 1) What is the genetic architecture of water use physiology traits in locally adapted A. thaliana? 2) How plastic are these water use physiology traits? and 3) Do water use physiology QTL colocalize with fitness QTL measured in the field?

\section{Material and methods}

\subsection{Environmental parameters in the field}

In order to further quantify the environmental differences between the field sites in Sweden and Italy we set up environmental sensors at the local sites and measured two soil characteristics relevant to drought stress, water potential and volumetric water content. These measurements were recorded once every hour, 24 hours a day using 5TM Soil Moisture and Temperature Sensor, Decagon Devices ${ }^{\circledR}$ (installed $1.5 \mathrm{~cm}$ below ground). Each environmental parameter was recorded using Em50 ${ }^{\circledR}$ Data Loggers (Decagon Devices Inc.) from 2010-2012.

To determine drought-related environmental parameters at a larger time scale, we obtained publicly available climatic parameters describing the Italy and Sweden field locations. First, we accessed historic climate data for monthly maximum and minimum temperature and average precipitation from WorldClim at 30-second arc resolution [27]. Additionally, we obtained data detected by NOAA's satellite based Advanced Very High Resolution Radiometer. These were measurements of the smoothed Normalized 
Difference Vegetation Index (NDVI) and the NDVI derived Vegetative Health Index (VHI), a proxy for detecting drought conditions. VHI values below 40 were considered to indicate drought conditions [28]. These NDVI and VHI data have been collected weekly since 1981 at $16 \mathrm{~km}^{2}$ resolution and were downloaded from (ftp://ftp.star.nesdis.noaa.gov/pub/corp/scsb/wguo/data/VHP_16km/VH). Summary statistics for weekly NDVI values were calculated to visualize the ecosystem wide growing season throughout the year. To assess the historic occurrence of drought by week, we binned drought conditions determined from VHI data into mild $(30<\mathrm{VHI}<40)$, moderate $(20<\mathrm{VHI}<30)$, severe $(10<\mathrm{VHI}<20)$, and extreme $(0<\mathrm{VHI}<10)$, and calculated the frequency of each weekly since 1981 at home locations of the Italy and Sweden ecotypes. Lastly, we calculated weekly photoperiod in each year based on latitude using the 'geosphere' package in R [27].

\subsection{Mapping population and RIL genotyping}

We used the mapping population described in Ågren et al. (2013, [20]), which was developed from locally adapted parental populations of $A$. thaliana. The two parents were collected near the northern (Rodasen, Sweden; 62 $48^{\prime} \mathrm{N} 18^{\circ} 12^{\prime} \mathrm{E}$ ) and southern limits (Castelnuovo de Porto, Italy: $42^{\circ} 07^{\prime} \mathrm{N} 12^{\circ} 29^{\prime} \mathrm{E}$ ) of the native geographic range of the species. Recombinant inbred lines (RILs) were derived from nine generations of selfing and single seed descent of the $\mathrm{F}_{1}$ hybrid of the parental lines. Agren and colleagues (2013, [20]) reported QTL for fitness (390 RILs) in the field over three seasons, which we used to compare to our results on plant water use physiology. Our experiment used $374 \mathrm{~F}_{9}$ RILs from this cross between Sweden (SW, sire) and Italy (IT, dam) genotypes. We excluded 16 RILs that Ågren and colleagues (2013) used in the field because of low seed availability or poor germination in the greenhouse.

To conduct QTL mapping, we used the published genotypes of the A. thaliana Sweden x Italy RILs. The complete description of the parental re-sequencing, RIL genotyping platform and construction of linkage map can be found in Ågren et al. (2013, [20]). Briefly, SNPs were identified from re-sequencing of the parents (Illumina GAII platform; 100-bp paired-end reads; Bowtie alignment using A. thaliana reference 
sequence Columbia, TAIR v9). RILs were genotyped using 384 single nucleotide polymorphisms (SNPs) via the Golden Gate Assay (Illumina®).

\subsection{Pre-drydown growth conditions}

Plants were grown in 2.5 " $(140 \mathrm{~mL})$ Kord $^{\circledR}$ pots lined with polyester cloth and filled with fritted clay (Profile Porous Ceramic Greens Grade ${ }^{\circledR}$ ) due to its more predictable and repeatable moisture release properties than regular potting soil [29-31]. We planted five seeds per pot (32 pots per flat) and arranged the plants in a split-plot design using three growth chambers (Conviron ATC60, Controlled Environments, Winnipeg, MB) as blocks, with two replicates per block. Seeds were stratified at $4^{\circ} \mathrm{C}$ for five days in growth chambers. After stratification, the growth chamber conditions were set to 13 -hour nights at $10^{\circ} \mathrm{C}, 11$-hour days at $18^{\circ} \mathrm{C}$, with a light intensity of approximately $330 \mu \mathrm{mol}$ photons $\mathrm{m}^{-2} \mathrm{sec}^{-1}$ Photosynthetic Photon Flux Density. Sweden and Italy parents were each replicated 30 times while the 379 RILs were replicated six times for a total of 2,304 pots. All plants germinated within two to three days following stratification. Each pot was thinned to a single seedling fourteen days after stratification. We fertilized the growing seedlings once a week using $0.5 x$ Hoagland's solution.

\subsection{Progressive dry down and phenotyping}

After 42 days post-stratification, we began a progressive drydown in half of the replicates of each genotype (dry treatment), while we continued watering the other half of the replicates to saturation (wet treatment). We assigned the dry treatment to 12 out of the 24 flats in each chamber. Our goal for the dry treatment was to simulate a slow-onset drought, which we accomplished by adjusting the gravimetric water content of individual pots on a daily basis in a progressive manner (from 95\%, 90\%, 80\%, 75\%, 70\%, 65\%, $60 \%, 55 \%, 50 \%$, to $40 \%$ saturation or target water content). This was performed by weighing each pot and comparing the weight to saturated and dry weights for the given pot, determined before planting. The amount of water added to each pot was then adjusted to reach the target water content. This plant-specific water adjustment is an important feature of our experimental design because we wanted to account for the 
significant, yet usually unaccounted for, variation in the rate of water consumption among plants (Figure S1).

At the end of the treatment period we harvested all plants, measuring a set of drought-related traits in both wet and dry treatments. We weighed above ground biomass to determine leaf water content (LWC $=$ (wet biomass - dry biomass $) \div$ wet biomass $)$. We also scanned the rosette to determine the projected rosette area (RA). RA was calculated using the scanned images in Image $\mathbf{J}$ [32] calibrated to the nearest square millimeter. We then lyophilized the harvested tissue and measured dry rosette weight (DRW). Finally, we conducted stable isotope analyses using an elemental analyzer interfaced to a continuous flow isotope ratio mass spectrometer at the UC-Davis Stable Isotope Facility. Lyophilized leaf tissue samples (two milligrams per sample) from each genotype were analyzed to determine the carbon isotope ratio $\left(\delta^{13} \mathrm{C}\right.$, expressed relative to the Vienna PeeDee Belemnite), and the percent mass of nitrogen in the leaves.

\subsection{Quantitative genetics and QTL analysis}

We performed all quantitative genetic analyses of phenotypes using $\mathrm{JMP}^{\circledR} \mathrm{v} 10$ (SAS Corporation). Using data for just the Recombinant Inbred Lines, for each RIL $\mathrm{x}$ treatment combination, we estimated breeding values using the least square means of each water use physiology trait $\left(\delta^{13} \mathrm{C}, \mathrm{RA}, \mathrm{DRW}, \mathrm{LWC}\right.$, and $\left.\% \mathrm{~N}\right)$ from mixed-model ANOVA using restricted maximum likelihood method (REML) with unbounded variance components. For each trait, we fit a model with treatment as fixed effect and genotype, genotype by treatment and flats nested within chamber (specific growth chamber where plants were grown) as random effects. To estimate environment-specific broad sense heritability of each trait, we used the same model excluding treatment since we evaluated the model within wet and dry treatment. To estimate variance explained by each factor in the model, we treated genotype, environment (wet or dry treatment), and genotype by environment as random effects. After checking for significant outliers and normality of the variables, we performed Pearson correlation of the breeding values (least square means) between all pairs of traits to estimate the genetic correlation.

We performed QTL mapping using R/qtl [33]. We used Haley-Knott regression to determine the appropriate number of QTL and their interactions for each water use 
physiology trait. For each trait $\left(\delta^{13} \mathrm{C}, \mathrm{RA}, \mathrm{DRW}, \mathrm{LWC}\right.$, and $\left.\% \mathrm{~N}\right)$, we mapped QTL for wet treatment, dry treatment. In addition we mapped plasticity using the difference in the breeding value of each RIL between wet-dry treatments. This character state approach of assessing plasticity has been described by Via and colleagues (1995, [34]). To calculate the significance threshold for additive and epistatic QTL, we performed 10,000 permutations to control for a genome-wide error rare of 0.05 [35]. We determined the best model that incorporates both additive and epistatic interaction through stepwise selection [36]. Genotypic effect sizes (in units of the traits) for each QTL were calculated from regression coefficients from the best multiple regression model using the fitqtl procedure [33]. For each QTL, we also calculated 95\% credible intervals around each point estimate.

From the QTL analyses in the two treatments we identified two categories of QTL: inducible and constitutive (sensu Monforte et al. 1997 [37]). Two overlapping QTLs (determined by point estimates within each other's 95\% credible intervals) that were detected in the two treatments were constitutive, while QTLs that were only detected in wet or dry treatment were inducible.

To identify overlap of water use physiology QTL with fitness QTL, we compared the position of QTL identified in this study and the intervals that contain fitness QTL (as described in Ågren et al. 2013 [20]). These intervals represent the range of point estimates of fitness QTL observed in more than one site and year combination (Sweden or Italy; 2009-2011). We determined colocalization between trait and fitness QTL if the point estimate of the water use physiology QTL was within the fitness QTL interval (for a full description, see Ågren et al. 2013 [20]).

\subsection{Identification of candidate genes}

To identify the potential candidate genes under adaptive QTL, we identified all the genes in the QTL intervals using the A. thaliana annotated genome TAIR 9 (https://www.arabidopsis.org/). We calculated the number of SNPs and the proportion of nonsynonymous changes between the Italy and Sweden parental lines using the Perl script 'Arabidopisis_compare_genomes' by David Lowry (github citation: https://github.com/davidbryantlowry/Arabidopsis_compare_genomes). We then selected 
genes with at least one predicted amino acid difference between the Italian and Swedish

parents, based on 56,618 nonsynonymous SNPs identified between the parental lines. In order to interpret the biological meaning of the list of divergent genes within the colocalizing fitness-water use physiology QTL, we used Gene Set Enrichment Analysis in Agri-GO (http://bioinfo.cau.edu.cn/agriGO/). We used the A. thaliana Col-0 genome (v9) as the background reference. We determined significant enrichment using Fisher's Exact test and corrected for multiple testing using Benjamini and Hochberg method (1995, [38]) at False Discovery Rate of $\alpha=0.05$.

\section{Results}

\subsection{Comparison of drought-related environmental parameters between Italy and Sweden sites}

Across three years and in each period of A. thaliana growth, on average, there was significantly more water (volumetric water content) in the soil in Sweden than in Italy, although this was most dramatic in the inter-season period when the plants were not growing (contrasts in ANOVA, $\mathrm{df}=1, \mathrm{P}<0.05$, Table 1; see Fig. 1 for details of growing seasons). Excluding inter-season, the germination period showed the largest mean difference $\left(0.02 \mathrm{~m}^{3} / \mathrm{m}^{3}\right)$ in soil water content between the two sites. Although water content during flowering was also on average higher in Sweden, the water potential, a measure of the availability of water to the plants, was actually more negative.

Furthermore, in all three years, there was a larger range of water potential during the growing season in Sweden (Table 2), indicating larger drying events.

Longer-term climate data supported the observation of the Swedish site as drier during the growing season. Precipitation was lower during the growing season at the Sweden site (Fig. 1E). Combined with longer photoperiods, this leads to a greater frequency of mild and moderate drought at the Sweden site, as corroborated by the vegetative health index (Fig. 1B).

\subsection{Water use physiology traits of parents and RILs}

While the water use efficiency $\left(\delta^{13} \mathrm{C}\right)$ of the two parents did not differ in the wet treatment, we found that the Swedish genotype had significantly higher water use 
efficiency (less negative $\delta^{13} \mathrm{C}$ ) than the Italian genotype in the dry treatment (Table 3). Swedish parents had significantly larger rosette area in the wet but not in the dry treatment. Dry rosette weight was significantly higher in the Swedish parent in both wet and dry treatment. We found no significant difference in leaf water content between the two parents in the wet treatment. Similarly, we found no significant difference in percent leaf nitrogen in the wet treatment or the dry treatment. Interestingly, we found that in all traits except percent leaf nitrogen, only the Swedish parent showed a significant change in the mean trait value between wet and dry treatment (Student's $t$ LS means difference, $\alpha$ $=0.05)$.

All water use physiology traits showed transgressive segregation in both wet and dry treatment. Three water use physiology traits $\left(\delta^{13} \mathrm{C}\right.$, rosette area, and dry rosette weight) exhibited larger range of mean genotypic values in the wet than in the dry treatment while leaf water content showed an opposite trend. The range of phenotypic values was approximately the same for leaf nitrogen between wet and dry treatments (Figure S2).

\subsection{Genotype by environment interaction (GxE) and heritability}

We found highly significant $(\mathrm{P}<0.001)$ main effects of genotype and environment for all traits. Table 4 shows the significant GxE interactions $(P<0.05)$ for rosette area and dry rosette weight. Broad sense heritability $\left(\mathrm{H}^{2}\right)$ was generally higher in the wet treatment than in the dry treatment (four out of five traits), except for $\delta^{13} \mathrm{C}$ where we found an opposite trend. Leaf water content in the dry treatment was the least heritable trait $\left(\mathrm{H}^{2}=21.07 \%\right)$, while $\delta^{13} \mathrm{C}$ showed the highest broad sense heritability at $48.5 \%$.

\subsection{Genetic correlations among water use physiology traits}

We found significant correlations $\left(r_{G}\right)$ among the water use physiology traits between wet and dry treatments (Figure 2). Water use efficiency, estimated by $\delta^{13} \mathrm{C}$ had a significant positive correlation with leaf water content $\left(r_{G}=0.24^{\delta 13 \mathrm{C} \text {-wet }}\right.$ and $\left.0.26^{\delta 13 \mathrm{C} \text {-dry }}\right)$ and $\%$ leaf nitrogen $\left(\% \mathrm{~N}, r_{G}=0.44^{\delta 13 \mathrm{C} \text {-wet }}\right.$ and $\left.0.39^{\delta 13 \mathrm{C} \text {-dry }}\right)$. More water-use efficient genotypes (less negative $\delta^{13} \mathrm{C}$ ) had higher water content and retained more nitrogen. In 

wet and $\left.-0.39^{\delta 13 \mathrm{C}-d r y}\right)$ and dry rosette weight $\left(r_{G}=-0.40^{\delta 13 \mathrm{C}-\text { wet }}\right.$ and $\left.-0.39^{\delta 13 \mathrm{C}-\mathrm{dry}}\right)$. Genotypes that were more water use efficient had smaller rosettes and lower dry rosette weight. Rosette area was significantly positively correlated with dry rosette weight $\left(r_{G}=0.73^{\text {RA- }}\right.$ wet and $\left.0.69^{\text {RA-dry }}\right)$ but was negatively correlated with both leaf water content $\left(r_{G}=-0.52^{\text {RA- }}\right.$ wet and $\left.-0.45^{\text {RA-dry }}\right)$ and $\%$ leaf nitrogen $\left(r_{G}=-0.70^{\text {RA-wet }}\right.$ and $\left.-0.55^{\text {RA-dry }}\right)$. We found that genotypes that had higher water content also had higher leaf $\%$ nitrogen $\left(r_{G}=0.73^{\text {RA-wet }}\right.$ and $0.69^{\text {RA-dry }}$ ). All traits showed the same trend in genetic correlation when analyzed within wet or dry treatment (Figure S3).

\subsection{QTL for water use physiology traits}

We detected a total of 72 quantitative trait loci (QTL) across five water use physiology traits (Table 5). These included 32 QTL found in the wet and 31 QTL in the dry treatment. Additionally, we found nine plasticity QTL, which were mapped from the phenotypic difference between environments. Interestingly, many of these trait QTL were found in both treatments (i.e., constitutive; Table 5, Figure 3).

We found six genomic regions where water use physiology and fitness QTL colocalized with fitness (Figure 3). We also observed two hotspot regions where multiple water use physiology QTL colocalized with fitness QTL. These hotspots of colocalization occurred on Chromosome (henceforth, Chr) II and III.

In this section, we present the QTL analysis results for each trait. The QTL nomenclature is as follows: each trait $\left(\delta^{13} C, R A, D R W, L W C\right.$, and $\left.\% N\right)$ is preceeded by a letter $w$ (wet treatment), $d$ (dry treatment), or $p$ (plasticity); followed by two numbers, chromosome number and centimorgan position. For example, $w \delta^{13} C 4: 31$, is a $\delta^{13} C$ QTL identified in the wet treatment. This QTL is located in chromoromse 4 at position $31 \mathrm{cM}$.

3.5.1. $\delta^{13} C$. There were a total of eight QTL in the dry treatment and nine QTL in the wet treatment. Combined, these QTL explained a total of $45.32 \%$ and $56.77 \%$ of $\delta^{13} \mathrm{C}$ variation in dry and wet treatments respectively. In both treatments, there were five QTL where the Swedish genotype increased $\delta^{13} \mathrm{C}$ (less negative thus more water use efficient). There were seven constitutive QTL while a total of three QTL were inducible $\left(w \delta^{13} C\right.$ 
4:31, $d \delta^{13} C 4: 11$, and $\left.d \delta^{13} C 4: 17\right)$. We detected two plasticity QTL ( $p \delta^{13} C 3: 14$ and $p \delta^{13} C 4: 39$ ), which explained a total $6.75 \%$ of the phenotypic variation for the difference in $\delta^{13} \mathrm{C}$ between wet and dry treatments. One of this plasticity QTL, $p R A$ 3:16, colocalized with a fitness QTL on Chr III. In addition, two constitutive but no inducible QTL colocalized with fitness QTL.

3.5.2. Rosette area $(R A)$. We detected nine and seven QTL in the wet and dry treatment, respectively. In total these QTL explained a total of $39.43 \%$ (wet) and $33.7 \%$ (dry) of the variation in rosette area. In the majority of the cases (9 out of 16 QTL), the Swedish genotype decreased rosette area. We found six constitutive QTL for rosette area. In addition, there were three inducible QTL ( $w R A$ 2:22, $w R A$ 3:57, and $d R A 4: 14$ ). There were three plasticity QTL that explained a total of $16.4 \%$ of the variation in the plasticity on rosette area. Two of this plasticity QTL, $p R A$ 2:59 and $p R A$ 3:16, colocalized with fitness QTL. In addition, we found other QTL that colocalized with fitness: three constitutive QTL, and one drought-inducible QTL.

3.5.3. Dry rosette weight ( $D R W$ ). Six QTL explained a total of $38.93 \%$ of the phenotypic variation in the wet treatment while four QTL explained a total of $25.82 \%$ of the phenotypic variation in the dry treatment. In 4/10 of the QTL, the Sweden genotype reduced dry rosette weight. We detected four constitutive QTL and one inducible QTL. For plasticity in DRW, we found three QTL that explained $16.68 \%$ of the variation in the difference between wet and dry treatment. Of these three plasticity QTL, only one ( $p D R W 2: 55$ ) colocalize with fitness. Furthermore, two constitutive and one wetinducible QTL colocalized with fitness.

3.5.4. Leaf water content $(L W C)$. We found four QTL in the wet treatment and five QTL in the dry treatment, which in total explained $23.23 \%$ (wet) and $23.18 \%$ (dry) of the phenotypic variation in leaf water content. In 5/9 QTL across the two treatments, the effect of a QTL was such that the substitution of the Swedish allele decreased leaf water content. We did not find any plasticity QTL for this trait. We found three constitutive 
QTL, and three inducible QTL for leaf water content. Only one of the constitutive QTL and no inducible QTL colocalized with fitness.

3.5.5. Percent leaf nitrogen $(\% N)$. A total of 11 QTL, five from wet and six from dry treatment, explained a total of $30.39 \%$ (wet) and $32.91 \%$ (dry) of the phenotypic variation in percent leaf nitrogen. In 6/11 QTL, the Swedish genotype decreased the amount of nitrogen stored in the leaf. A total of five constitutive QTL and only one drought-inducible QTL were detected for this trait. We found a single $\% \mathrm{~N}$ plasticity QTL, $p \% N 4: 25$, which did not colocalize with fitness QTL. Three constitutive but no inducible QTL colocalized with fitness QTL.

\subsection{Candidate genes}

Water-use physiology and fitness QTL colocalized on all chromosomes, except on Chr IV. These putatively adaptive regions contained a total of 4,758 genes, $54 \%$ of which showed at least one amino acid difference (divergent) between the Italian and Swedish parent. Gene Set Enrichment Analysis (Fisher's exact test, FDR adjusted pvalue $<0.05)$ revealed that this list of potentially adaptive genes was enriched with factors involved in multiple biological processes (e.g., lipid localization, post embryonic development, cellular carbohydrate metabolic process etc., Table S1). In addition, we found 101 genes (Table S2) from the list of putatively adaptive genes that were annotated under the following GO terms: GO:0009414 (response to water deprivation) and GO:0006970 (response to osmotic stress). Of these, we found 31 candidate genes were within the hotspot QTL region on Chr II and 21 candidate genes on Chr III (Table S2).

\section{Discussion}

The genetic basis of adaptation is typically complex, involving many phenotypic traits and their underlying genes. While progress has been made toward understanding the genetic polymorphisms that underlie putatively adaptive traits, seldom are studies able to directly determine if loci underlying physiological traits might be the same as loci underlying local adaptation (i.e. fitness) to the ancestral environments. Here, we used locally adapted $A$. thaliana lines to identify QTL for traits that influence water use 
physiology, including plasticity in such traits, compare these QTL to the location of fitness QTL, and identify candidate genes underlying adaptive water use physiological QTL. We found that there were few genomic loci that control water-use physiology. Most of the water use physiology QTL were constitutive in nature (i.e., they were detected in both wet and dry treatments, blue symbols in Figure 3), and a few were only detected in wet or dry treatments (inducible QTL as red symbols in Figure 3). We also found that constitutive QTL $(44 \%, 11 / 25)$ were more commonly found to be adaptive relative to inducible QTL $(20 \%, 3 / 15)$.

\subsection{Water availability in Sweden and Italy}

During the growing season, precipitation was lower, and the frequency of drought was historically higher at the Swedish site (Fig. 1B, E). Further, we found that water was less available (more negative water potential) and more variable, in Sweden compared to Italy (Table 2). Consistent with this, for the measured drought avoidance traits, the Swedish genotype but not the Italian genotype, showed significant response to water treatment (Table 3).

The difference between Italy and Sweden ecotypes in WUE under dry conditions may reflect divergence in drought adaptive strategies resulting from different historic selective regimes. High WUE is a drought response strategy associated with dehydration avoidance. In contrast, low WUE efficiency is correlated the fast growth necessary for drought escape [39-42]. Rapid development to escape drought is a fundamental strategy that determines fitness in environments where water varies at spatial and temporal scales $[23,43]$. Indeed, the normal flowering period in Italy occurs just prior to the typical onset of drought conditions (Figure 1B) and may come at the cost of lost growth potential during weeks with peak photoperiod (Figure 1C). This, and the significantly lower WUE in the Italian ecotype under dry conditions may indicate the evolution of an adaptive drought escape strategy. 


\subsection{Constitutive and inducible water use physiology QTL}

Our results show that most of the water use physiology QTL were constitutive in nature. Of the few inducible QTL, about half them were expressed only under dry conditions while the other half were detected only in the well-watered treatment. A recent meta-analyses of multi-population experiments on water use physiology in maize showed a similar trend in the distribution of QTL between drought inducible and well-watered inducible QTL [44]. In addition, the same meta-analysis identified genes underlying these constitutive and inducible QTL. Specifically they showed that constitutive QTL in maize were commonly involved in synthesis of osmo-regulators (e.g. Proline). In contrast, genes under drought-response QTL (protein kinases and transcription factors) play a more regulatory role in abiotic response pathways like the abscissic acid response pathway.

\subsection{Plasticity in water use physiology traits}

Plants have evolved morphological and physiological adaptations that allow them to maximize growth and reproduction in a heterogeneous environment $[1,24,45]$. To understand the genetic architecture of these adaptations, we can find associations of genotype and phenotype within an environment. When we have at least two environments where replicated genotypes are grown, we can assess the degree at which the norms of reaction differ among the genotypes. In this study, using a character state approach [34], we mapped nine genomic locations associated with plasticity in water use physiology traits.

The intensity of environmental stress varies considerably over the growing season. Therefore, it is not surprising that plasticity of water use physiology can be under selection in nature [46]. In this study, 4/9 plasticity QTL colocalize with fitness suggesting that plastic response in water use efficiency (via $\delta^{13} \mathrm{C}$ ) and degree of growth under water limiting conditions (via RA and DRW) maybe important in determining fitness in the field. Given our environmental measurements between Sweden and Italy (see discussion above), it was not surprising that in all of the plasticity QTL that colocalized with fitness, the Sweden allele was associated with greater difference between treatments. 
Breeding directly for plasticity in water use physiology is a promising but underutilized avenue for crop improvement. This is likely due to the complication and effort of getting accurate estimate of breeding values from multiple discrete environments. While challenging, aggregating and evaluating yield data from growing similar genotypes within multi-year and multi-field site framework in a drought/no drought condition has been successfully implemented [47]. A major conclusion from that study is that the Optimum AQUAmax hybrids (Dupont Pioneer) showed greater yield stability (vs. non-AQUAmax hybrids) on drought conditions with no yield penalty under permissive conditions. The results from this industry-scale evaluation suggest a great promise in directly incorporating plasticity and GxE in future breeding efforts for agricultural crops.

\subsection{Adaptive QTL, hotspots and candidate genes}

Colocalization of multiple QTL (e.g. "hotspots") may be caused by pleiotropic gene action. Of particular interest are cases where physiological QTL colocalize with fitness QTL in the field - “adaptive trait QTL”. Such QTL hotspots may be due to the adaptive evolution of genes that pleiotropically affect fitness through physiology. These hotspots may contain important regulatory genes that lack annotation as drought responsive [48].

One of our objectives was to find regions of the genome where fitness and water use physiology QTL colocalized. Our experimental set up is conducive to this kind of analysis because we used the same mapping population as in the field, where fitness has been measured directly [20]. This is critical to identifying adaptive trait QTL because we can assess if QTL for these phenotypes might underlie QTL for fitness in the environments in which these genotypes evolved. Previous work in A. thaliana has employed similar approach in linking fitness variation with trait variation that is usually difficult to measure in the field. Oakley and colleagues $(2014,[21])$ used this approach to investigate the genetic basis of freezing tolerance in growth chamber experiments, and determined the extent to which freezing tolerance QTL contribute to local adaptation and fitness trade-offs in the field. A similar approach was also used to investigate flowering time in the same study system [22]. It is interesting to note that neither of these studies 
found large effect trait QTL in the regions of fitness QTL on Chr II and III that represent the "hotspots" of water use physiology we report here. Aside from flowering related traits, fitness can be determined by size related traits. A rich body of literature has documented this relationship between size and fecundity in many species [49]. In our study, seven out of the 19 QTLs for size (rosette area) colocalize with fitness. It is however noteworthy, that most of these size-related QTLs do not colocalize with other water use physiology traits like $\delta^{13} \mathrm{C}$. When they do colocalize, it is under QTL hotspots where multiple traits map to the same location.

The rich genomic resource available in A. thaliana allowed us to identify candidate genes underlying putatively adaptive water use physiology and fitness QTL. We identified polymorphic genes involved in associated pathways to narrow a list of candidate genes. From a large list of 4,758 genes underlying water use physiology and fitness QTL, we identified a total of 52 candidate genes under the two water use physiology QTL hotspot regions that colocalize with fitness (Supplementary Table S2).

Within the adaptive QTL region on Chr II, we identified 31 candidate genes. One notable candidate was At2g38750, which a single amino acid substitution (1/319) in the coding DNA sequence is not predicted to result in a premature stop codon. Commonly known as annexin ANNAT4, this candidate gene is a member of a family of calcium and phospholipid binding proteins [50]. Its expression is altered in response to dehydration, as well as salt and temperature stress [51]. Recently, Huh and colleagues (2010, [52]) show that overexpression of this gene results in drought sensitivity only during long day conditions. During germination ANNAT4 mutants were hypersensitive to osmotic stress and the hormone ABA [53], and mutant plants have reduced water loss and increased drought tolerance [52]. For the adaptive region on Chr III, we identified 21 candidate genes. A notable candidate for this region was At3g19290 (divergence $=0.002$ ). At this gene, the Italian and Swedish parents differ by a single amino acid substitution (1/431). This gene is ABF4/AREB2, a bzip transcription factor that mediates ABA signaling, and affecting growth, transpiration and drought tolerance [54-56]. In a recent study, Garcia and colleagues $(2014,[57])$ showed that overexpression of ABF4 in potato (Solanum tuberosum) enhances tuberization without a growth penalty. Thus this candidate gene may have value for breeding enhanced agricultural crops. 
The research presented here combines the detailed functional knowledge from $A$. thaliana with an evolutionary approach to start to dissect the genetic basis of drought adaptation. We used a mapping population developed from locally adapted Swedish and Italian populations and measured trait variances and co-variances in water use physiology traits, and how these respond to drought treatment in controlled conditions. Our water use efficiency experiment was motivated by the fact that Swedish site is drier and has a higher frequency of drought relative to the Italian site. We then mapped QTL controlling these traits and their plasticity to drought. We found that most QTL for water use physiology is constitutive rather than inducible. The QTL that we found were then compared to published QTL underlying local adaptation, to identify water use physiology QTL that colocalize with QTL controlling fitness in native field sites. In this analysis we found that plasticity on water use physiology traits may have fitness consequences. In addition, multiple water use physiology traits colocalize with fitness suggesting that adaptation to the environment is a function of multiple traits. Finally, we used data from resequencing the parents to identify potentially functionally important polymorphisms that may underlie this variation in water use physiology and local adaptation. This analysis revealed 52 candidate genes, several of which (e.g., ANNAT4 and ABF4/AREB2), show a great prospect for future functional analysis within this species or in other crop breeding systems as a whole. The paucity of data that connects fieldevaluated fitness with drought-related traits limits our understanding of the immense role of physiology in local adaptation. Our research harnessed the genetic wealth of Arabidopsis to identify candidate water use physiology genes that matter in the field. This candidate gene knowledge generated from the model plant Arabidopsis will be an invaluable resource to inform breeding of the next generation of crop species that are economically drought responsive.

\section{Acknowledgements}

We thank Kelsi Grogan, Jennifer DeWoody, Courtney Gomola, Richard Fletcher, and Jacob Landis for their assistance in the greenhouse work. This research was supported by National Science Foundation Grant 1022202 to JKM. 


\section{References}

[1] G.L. Stebbins, Aridity as a stimulus to plant evolution, Am. Nat., 86 (1952) 33-44.

[2] T.E. Juenger, Natural variation and genetic constraints on drought tolerance, Current Opinion in Plant Biology, 16 (2013) 274-281.

[3] N.J. Kooyers, The evolution of drought escape and avoidance in natural herbaceous populations, Plant Sci, 234 (2015) 155-162.

[4] J. Xu, H. Lafitte, Y. Gao, B. Fu, R. Torres, Z. Li, QTLs for drought escape and tolerance identified in a set of random introgression lines of rice, TAG Theoretical and Applied Genetics, 111 (2005) 1642-1650.

[5] A. Blum, Drought resistance, water-use efficiency, and yield potential - are they compatible, dissonant, or mutually exclusive?, Aust J Agr Res, 56 (2005) 1159-1168.

[6] A. Blum, Drought resistance and its improvement, Plant Breeding for Water-Limited Environments, (2011) 53-152.

[7] M.M. Chaves, J.P. Maroco, J.S. Pereira, Understanding plant responses to drought from genes to the whole plant, Funct Plant Biol, 30 (2003) 239-264.

[8] B. Jongdee, S. Fukai, M. Cooper, Leaf water potential and osmotic adjustment as physiological traits to improve drought tolerance in rice, Field Crop. Res., 76 (2002) 153163.

[9] K. Yamaguchishinozaki, K. Shinozaki, A novel cis-acting element in an Arabidopsis gene is involved in responsiveness to drought, low temperature, or high-salt stress, Plant Cell, 6 (1994) 251-264.

[10] E.A. Bray, Genes commonly regulated by water-deficit stress in Arabidopsis thaliana, J. Exp. Bot., (2004) erh270.

[11] Y. Fujita, M. Fujita, R. Satoh, K. Maruyama, M.M. Parvez, M. Seki, K. Hiratsu, M. Ohme-Takagi, K. Shinozaki, K. Yamaguchi-Shinozaki, AREB1 is a transcription 
activator of novel ABRE-dependent ABA signaling that enhances drought stress tolerance in Arabidopsis, Plant Cell, 17 (2005) 3470-3488.

[12] R. Kawaguchi, T. Girke, E.A. Bray, J. Bailey-Serres, Differential mRNA translation contributes to gene regulation under non-stress and dehydration stress conditions in Arabidopsis thaliana, Plant Journal, 38 (2004) 823-839.

[13] J.A. Kreps, Y. Wu, H.-S. Chang, T. Zhu, X. Wang, J.F. Harper, Transcriptome changes for Arabidopsis in response to salt, osmotic, and cold stress, Plant Physiol., 130 (2002) 2129-2163.

[14] M. Seki, M. Narusaka, J. Ishida, T. Nanjo, M. Fujita, Y. Oono, A. Kamiya, M. Nakajima, A. Enju, T. Sakurai, M. Satou, K. Akiyama, T. Taji, K. Yamaguchi-Shinozaki, P. Carninci, J. Kawai, Y. Hayashizaki, K. Shinozaki, Monitoring the expression profiles of 7000 Arabidopsis genes under drought, cold and high-salinity stresses using a fulllength cDNA microarray, Plant Journal, 31 (2002) 279-292.

[15] K. Shinozaki, K. Yamaguchi-Shinozaki, M. Seki, Regulatory network of gene expression in the drought and cold stress responses, Current Opinion in Plant Biology, 6 (2003) 410-417.

[16] Y. Uno, T. Furihata, H. Abe, R. Yoshida, K. Shinozaki, K. Yamaguchi-Shinozaki, Arabidopsis basic leucine zipper transcription factors involved in an abscisic aciddependent signal transduction pathway under drought and high-salinity conditions, Proc. Natl. Acad. Sci. U. S. A., 97 (2000) 11632-11637.

[17] R. Kesari, J.R. Lasky, J.G. Villamor, D.L.D. Marais, Y.J.C. Chen, T.W. Liu, W. Lin, T.E. Juenger, P.E. Verslues, Intron-mediated alternative splicing of Arabidopsis P5CS1 and its association with natural variation in proline and climate adaptation, Proc. Natl. Acad. Sci. U. S. A., 109 (2012) 9197-9202.

[18] A. Manzaneda, P.J. Rey, J. Anderson, E. Raskin, C. Weiss-Lehman, T. MitchellOlds, Natural variation, plasticity and tradeoffs of drought tolerance-related traits in the Brachypodium distachyon (Poaceae) ploidy complex, Evolution, (2013) in review. 
[19] J.K. McKay, J.H. Richards, K.S. Nemali, S. Sen, T. Mitchell-Olds, S. Boles, E.A. Stahl, T. Wayne, T.E. Juenger, Genetics of drought adaptation in Arabidopsis thaliana Ii. Qtl analysis of a new mapping population, Kas-1 X Tsu-1, Evolution, 62 (2008) 30143026.

[20] J. Ågren, C.G. Oakley, J.K. McKay, J.T. Lovell, D.W. Schemske, Genetic mapping of adaptation reveals fitness tradeoffs in Arabidopsis thaliana, Proc Natl Acad Sci U S A, 110 (2013) 21077-21082.

[21] C.G. Oakley, J. Ågren, R.A. Atchison, D.W. Schemske, QTL mapping of freezing tolerance: links to fitness and adaptive trade-offs, Molecular Ecology, 23 (2014) 43044315.

[22] E.L. Dittmar, C.G. Oakley, J. Ågren, D.W. Schemske, Flowering time QTL in natural populations of Arabidopsis thaliana and implications for their adaptive value, Mol Ecol, 23 (2014) 4291-4303.

[23] L.A. Donovan, S.A. Dudley, D.M. Rosenthal, F. Ludwig, Phenotypic selection on leaf water use efficiency and related ecophysiological traits for natural populations of desert sunflowers, Oecologia, 152 (2007) 13-25.

[24] D.L. Des Marais, J.K. McKay, J.H. Richards, S. Sen, T. Wayne, T.E. Juenger, Physiological genomics of response to soil drying in diverse Arabidopsis accessions, Plant Cell, 24 (2012) 893-914.

[25] I.J. Wright, P.B. Reich, M. Westoby, D.D. Ackerly, Z. Baruch, F. Bongers, J. Cavender-Bares, T. Chapin, J.H.C. Cornelissen, M. Diemer, J. Flexas, E. Garnier, P.K. Groom, J. Gulias, K. Hikosaka, B.B. Lamont, T. Lee, W. Lee, C. Lusk, J.J. Midgley, M.L. Navas, U. Niinemets, J. Oleksyn, N. Osada, H. Poorter, P. Poot, L. Prior, V.I. Pyankov, C. Roumet, S.C. Thomas, M.G. Tjoelker, E.J. Veneklaas, R. Villar, The worldwide leaf economics spectrum, Nature, 428 (2004) 821-827.

[26] J. Hereford, A quantitative survey of local adaptation and fitness trade-offs, Am Nat, 173 (2009) 579 - 588. 
[27] R.J. Hijmans, S.E. Cameron, J.L. Parra, P.G. Jones, A. Jarvis, Very high resolution interpolated climate surfaces for global land areas, Int J Climatol, 25 (2005) 1965-1978.

[28] R.P. Singh, S. Roy, F. Kogan, Vegetation and temperature condition indices from NOAA AVHRR data for drought monitoring over India, International Journal of Remote Sensing, 24 (2003) 4393-4402.

[29] N.S. Hill, J.G. Pachon, C.W. Bacon, Acremonium coenophialum-mediated shortand long-term drought acclimation in tall fescue, Crop Science, 36 (1996) 665-672.

[30] C.A. Meisner, K.J. Karnok, Peanut root response to drought stress, Agron. J., 84 (1992) 159-165.

[31] C.H.M. Vanbavel, R. Lascano, D.R. Wilson, Water Relations of Fritted Clay, Soil Sci Soc Am J, 42 (1978) 657-659.

[32] C.A. Schneider, W.S. Rasband, K.W. Eliceiri, NIH Image to ImageJ: 25 years of image analysis, Nat Methods, 9 (2012) 671-675.

[33] K.W. Broman, S. Sen, A guide to QTL mapping with R/qtl, Springer, New York, 2009.

[34] S. Via, R. Gomulkiewicz, G. Dejong, S.M. Scheiner, C.D. Schlichting, P.H. Vantienderen, Adaptive phenotypic plasticity - consensus and controversy, Trends Ecol. Evol., 10 (1995) 212-217.

[35] G.A. Churchill, R.W. Doerge, Empirical threshold values for quantitative trait mapping, Genetics, 138 (1994) 963-971.

[36] A. Manichaikul, J.Y. Moon, S. Sen, B.S. Yandell, K.W. Broman, A model selection approach for the identification of quantitative trait loci in experimental crosses, allowing epistasis, Genetics, 181 (2009) 1077-1086. 
[37] A.J. Monforte, M.J. Asin, E.A. Carbonell, Salt tolerance in Lycopersicon species IV. Genotype-by-salinity intertaction in quantitative trait loci detection: constitutive and response QTLs, Theor Appl Genet, 95 (1997) 706-713.

[38] Y. Benjamini, Y. Hochberg, Controlling the false discovery rate - a practical and powerful approach to multiple testing, J Roy Stat Soc B Met, 57 (1995) 289-300.

[39] R.S. Fletcher, J.L. Mullen, A. Heiliger, J.K. McKay, QTL analysis of root morphology, flowering time, and yield reveals trade-offs in response to drought in Brassica napus, J. Exp. Bot., 66 (2015) 245-256.

[40] J.K. Kelly, L.M. Holeski, H.S. Arathi, The genetic correlation between flower size and water use efficiency in monkeyflowers, Evol. Ecol. Res., 10 (2008) 147-152.

[41] A.M. Kenney, J.K. McKay, J.H. Richards, T.E. Juenger, Direct and indirect selection on flowering time, water-use efficiency (WUE, delta C-13), and WUE plasticity to drought in Arabidopsis thaliana, Ecol Evol, 4 (2014) 4505-4521.

[42] J.K. McKay, J.H. Richards, T. Mitchell-Olds, Genetics of drought adaptation in Arabidopsis thaliana: I. Pleiotropy contributes to genetic correlations among ecological traits, Molecular Ecology, 12 (2003) 1137-1151.

[43] J.P. Mojica, J.K. Kelly, Viability selection prior to trait expression is an essential component of natural selection, Proceedings of the Royal Society B-Biological Sciences, 277 (2010) 2945-2950.

[44] Z.F. Hao, X.H. Li, X.L. Liu, C.X. Xie, M.S. Li, D.G. Zhang, S.H. Zhang, Metaanalysis of constitutive and adaptive QTL for drought tolerance in maize, Euphytica, 174 (2010) 165-177.

[45] T.E. Juenger, J.K. Mckay, N. Hausmann, J.J.B. Keurentjes, S. Sen, K.A. Stowe, T.E. Dawson, E.L. Simms, J.H. Richards, Identification and characterization of QTL underlying whole-plant physiology in Arabidopsis thaliana: delta C-13, stomatal conductance and transpiration efficiency, Plant Cell Environ, 28 (2005) 697-708. 
[46] M.S. Heschel, A. Evankow, K.B. Wolfson, J.E. Carlson, K.E. Holsinger, Drought response diversification in African protea species, Int. J. Plant Sci., 175 (2014) 442-449.

[47] J. Gaffney, J. Schussler, C. Loffler, W.G. Cai, S. Paszkiewicz, C. Messina, J.

Groeteke, J. Keaschall, M. Cooper, Industry-scale evaluation of maize hybrids selected for increased yield in drought-stress conditions of the US corn belt, Crop Science, 55 (2015) 1608-1618.

[48] R. Breitling, Y. Li, B.M. Tesson, J.Y. Fu, C.L. Wu, T. Wiltshire, A. Gerrits, L.V. Bystrykh, G. de Haan, A.I. Su, R.C. Jansen, Genetical genomics: spotlight on QTL hotspots, Plos Genetics, 4 (2008).

[49] J.G. Kingsolver, H.E. Hoekstra, J.M. Hoekstra, D. Berrigan, S.N. Vignieri, C.E. Hill, A. Hoang, P. Gibert, P. Beerli, The strength of phenotypic selection in natural populations, Am. Nat., 157 (2001) 245-261.

[50] G.B. Clark, A. Sessions, D.J. Eastburn, S.J. Roux, Differential expression of members of the annexin multigene family in Arabidopsis, Plant Physiol., 126 (2001) 1072-1084.

[51] A. Cantero, S. Barthakur, T.J. Bushart, S. Chou, R.O. Morgan, M.P. Fernandez, G.B. Clark, S.J. Roux, Expression profiling of the Arabidopsis annexin gene family during germination, de-etiolation and abiotic stress, Plant Physiol Biochem, 44 (2006) 13-24.

[52] S.M. Huh, E.K. Noh, H.G. Kim, B.W. Jeon, K. Bae, H.C. Hu, J.M. Kwak, O.K. Park, Arabidopsis annexins AnnAt1 and AnnAt4 interact with each other and regulate drought and salt stress responses, Plant Cell Physiol, 51 (2010) 1499-1514.

[53] S. Lee, E.J. Lee, E.J. Yang, J.E. Lee, A.R. Park, W.H. Song, O.K. Park, Proteomic identification of annexins, calcium-dependent membrane binding proteins that mediate osmotic stress and abscisic acid signal transduction in Arabidopsis, Plant Cell, 16 (2004) 1378-1391.

[54] J.Y. Kang, H.I. Choi, M.Y. Im, S.Y. Kim, Arabidopsis basic leucine zipper proteins that mediate stress-responsive abscisic acid signaling, Plant Cell, 14 (2002) 343-357. 
[55] T. Yoshida, Y. Fujita, K. Maruyama, J. Mogami, D. Todaka, K. Shinozaki, K. Yamaguchi-Shinozaki, Four Arabidopsis AREB/ABF transcription factors function predominantly in gene expression downstream of SnRK2 kinases in abscisic acid signalling in response to osmotic stress, Plant Cell Environ, 38 (2015) 35-49.

[56] T. Yoshida, Y. Fujita, H. Sayama, S. Kidokoro, K. Maruyama, J. Mizoi, K. Shinozaki, K. Yamaguchi-Shinozaki, AREB1, AREB2, and ABF3 are master transcription factors that cooperatively regulate ABRE-dependent ABA signaling involved in drought stress tolerance and require ABA for full activation, The Plant journal : for cell and molecular biology, 61 (2010) 672-685.

[57] M.N.M. Garcia, M. Stritzler, D.A. Capiati, Heterologous expression of Arabidopsis ABF4 gene in potato enhances tuberization through ABA-GA crosstalk regulation, Planta, 239 (2014) 615-631. 
Table 1. Contrast of field-measured water potential and water content between Italy and Sweden across three years (2010-2013). Asterisk (*) indicates the site that has a significantly higher water potential or water content. Columns are growth period of $A$. thaliana(period), site, number of observation $(\mathrm{N})$, arithmetic mean (mean), standard error (SE).Germination period: Italy (It) Oct 15-Nov 15, Sweden (Sw) Aug 20- Sept 20. Growth period: (It) Nov 16- Feb 14, (Sw) Sept 21- Apr 30, Flowering period (It) Feb 15Mar 15, (Sw) May 1- June 1. Inter-season period: (It) Mar 16- Oct 14, (Sw) June 2- Aug 19.

\begin{tabular}{llllllll}
\hline \multirow{2}{*}{ Period } & Site & \multicolumn{2}{l}{ Water content $\left(\mathrm{m}^{3} / \mathbf{m}^{3}\right)$} & \multicolumn{3}{c}{ Water potential (MPa) } \\
\cline { 3 - 7 } & & $\mathbf{N}$ & Mean & SE & N & Mean & SE \\
\hline Germination & Italy & 9129 & 0.1931 & 0.0005 & 6218 & -0.039 & 0.001 \\
& Sweden & 9216 & $0.2129^{*}$ & 0.0004 & 6139 & $-0.023^{*}$ & 0.001 \\
\multirow{2}{*}{ Growth } & Italy & 30253 & 0.1920 & 0.0002 & 21840 & -0.020 & 0.000 \\
& Sweden & 94165 & $0.1945^{*}$ & 0.0002 & 62816 & -0.021 & 0.000 \\
\multirow{2}{*}{ Flowering } & Italy & 9464 & 0.1870 & 0.0006 & 7053 & $-0.028^{*}$ & 0.001 \\
& Sweden & 13824 & $0.1928^{*}$ & 0.0005 & 9216 & -0.057 & 0.001 \\
\multirow{2}{*}{ Inter-season } & Italy & 48726 & 0.1230 & 0.0003 & 34118 & -0.240 & 0.001 \\
& Sweden & 26025 & $0.1611^{*}$ & 0.0003 & 17350 & $-0.127^{*}$ & 0.001 \\
\hline
\end{tabular}


Table 2. Range and minimum field soil water potential $(\mathrm{kPa})$ between Sweden and Italy across three years (2010-2012). Asterisk (*) indicates the site that has significantly higher range in water potential. The range and min are determined during the growth period in each site.

\begin{tabular}{llrlr}
\hline Year & Site & Range & & Min \\
\hline 2010 & Italy & 466.00 & & -477.00 \\
& Sweden & 820.00 & $*$ & -830.00 \\
2011 & Italy & 89.00 & & -100.00 \\
& Sweden & 272.00 & $*$ & -281.00 \\
2012 & Italy & 7.00 & & -18.00 \\
& Sweden & 255.00 & $*$ & -263.00 \\
\hline
\end{tabular}


Table 3. Parental least square means of drought tolerance traits on wet and dry conditions.

\begin{tabular}{|c|c|c|c|c|c|c|c|}
\hline Treatment & Trait & Parent & $\mathbf{N}$ & LSM & SE & F-ratio & $P$ \\
\hline \multirow{10}{*}{ Wet } & \multirow[t]{2}{*}{$\delta^{13} \mathrm{C}$} & $\overline{I t a l y}$ & 15 & -29.56 & 0.3394 & \multirow[t]{2}{*}{0.426} & \multirow[t]{2}{*}{$\overline{0.519}$} \\
\hline & & Sweden & 15 & -29.70 & 0.3395 & & \\
\hline & \multirow[t]{2}{*}{ RA } & Italy & 15 & 1961.30 & 158.1836 & \multirow[t]{2}{*}{4.946} & \multirow[t]{2}{*}{0.035} \\
\hline & & Sweden & 15 & 2231.65 & 158.1113 & & \\
\hline & \multirow[t]{2}{*}{ DRW } & Italy & 15 & 61.77 & 7.6861 & \multirow[t]{2}{*}{9.993} & \multirow[t]{2}{*}{0.004} \\
\hline & & Sweden & 15 & 83.82 & 7.6835 & & \\
\hline & \multirow[t]{2}{*}{ LWC } & Italy & 15 & 0.90 & 0.0004 & \multirow[t]{2}{*}{0.138} & \multirow[t]{2}{*}{0.713} \\
\hline & & Sweden & 15 & 0.91 & 0.0004 & & \\
\hline & \multirow[t]{2}{*}{$\% \mathrm{~N}$} & Italy & 15 & 5.12 & 0.2071 & \multirow[t]{2}{*}{2.029} & \multirow[t]{2}{*}{0.165} \\
\hline & & Sweden & 15 & 4.79 & 0.2070 & & \\
\hline \multirow[t]{10}{*}{ Dry } & \multirow[t]{2}{*}{$\delta^{13} \mathrm{C}$} & Italy & 14 & -29.42 & 0.1924 & \multirow[t]{2}{*}{4.464} & \multirow[t]{2}{*}{0.044} \\
\hline & & Sweden & 14 & -29.11 & 0.1923 & & \\
\hline & \multirow[t]{2}{*}{ RA } & Italy & 14 & 1743.96 & 135.0163 & \multirow[t]{2}{*}{0.017} & \multirow[t]{2}{*}{0.897} \\
\hline & & Sweden & 15 & 1735.88 & 132.7830 & & \\
\hline & \multirow[t]{2}{*}{ DRW } & Italy & 14 & 45.56 & 4.1213 & \multirow[t]{2}{*}{10.303} & \multirow[t]{2}{*}{0.003} \\
\hline & & Sweden & 15 & 58.00 & 6.6529 & & \\
\hline & \multirow[t]{2}{*}{ LWC } & Italy & 14 & 0.90 & 0.0069 & \multirow[t]{2}{*}{2.697} & \multirow[t]{2}{*}{0.112} \\
\hline & & Sweden & 15 & 0.89 & 0.0069 & & \\
\hline & \multirow[t]{2}{*}{$\% \mathrm{~N}$} & Italy & 14 & 4.37 & 0.2160 & \multirow[t]{2}{*}{2.348} & \multirow[t]{2}{*}{0.138} \\
\hline & & Sweden & 14 & 4.14 & 0.2159 & & \\
\hline
\end{tabular}


Table 4. Percent variation explained and broad sense heritability of water use physiology traits of Sweden x Italy RILs in A. thaliana. Environment represents the wet and dry treatment from the progressive drydown experiment of recombinant inbred lines. Superscripts represent $P$-value $<0.001(* *),<0.05(*)$, and $>0.05\left({ }^{\mathrm{ns}}\right)$.

\begin{tabular}{|c|c|c|c|c|c|}
\hline \multirow{2}{*}{ Traits } & \multicolumn{3}{|c|}{ Percent variation explained by a model parameter } & \multicolumn{2}{|c|}{ Heritability } \\
\hline & Genotype $(\mathrm{G})$ & Environment (E) & $\mathrm{G} \times \mathrm{E}$ & Wet & Dry \\
\hline$\delta^{13} \mathrm{C}$ & $45.42 * *$ & $7.81 * *$ & $1.17^{\mathrm{ns}}$ & 47.39 & 48.45 \\
\hline Rosette area & $36.26 * *$ & $11.35 * *$ & $3.69 *$ & 46.97 & 37.12 \\
\hline Dry rosette weight & $36.70 * *$ & $13.21 * *$ & $3.26^{*}$ & 47.19 & 36.29 \\
\hline Leaf water content & $20.12 * *$ & $12.15 * *$ & $1.37^{\mathrm{ns}}$ & 25.69 & 21.07 \\
\hline Percent nitrogen & $29.08 * *$ & $27.58 * *$ & $1.52^{\mathrm{ns}}$ & 46.35 & 35.17 \\
\hline
\end{tabular}




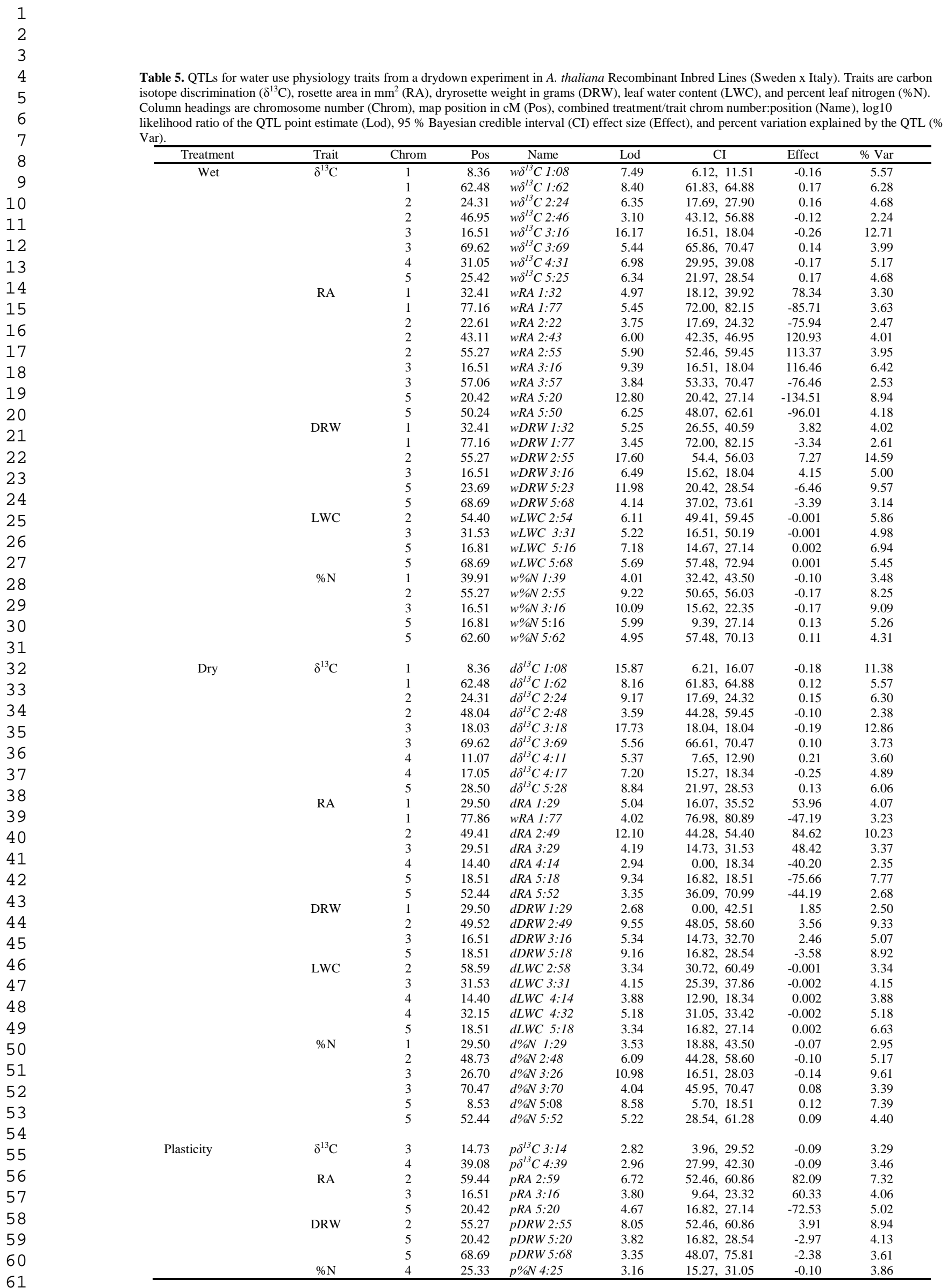




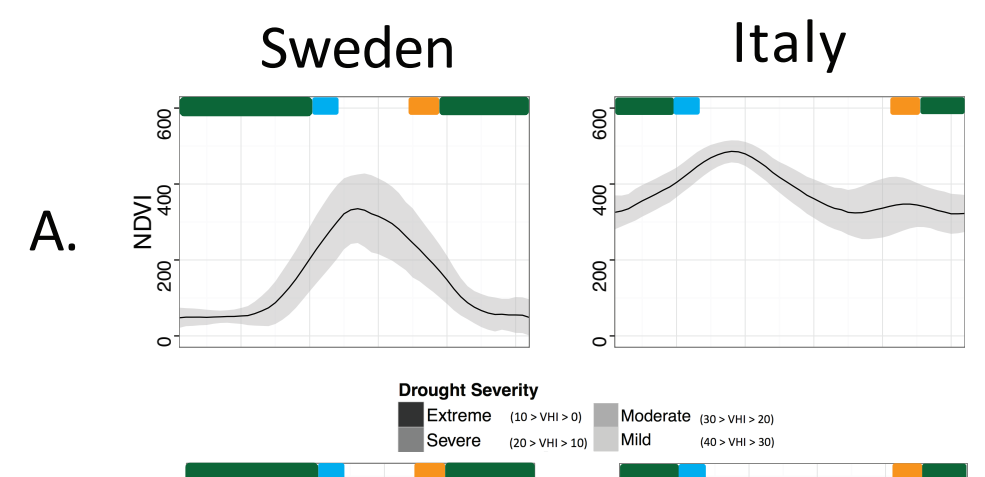

B.
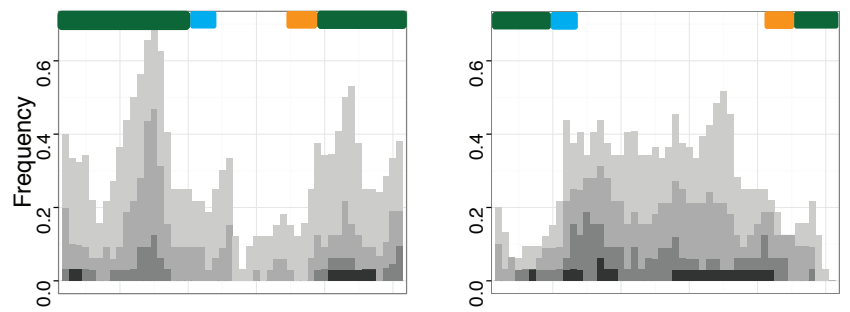

C.
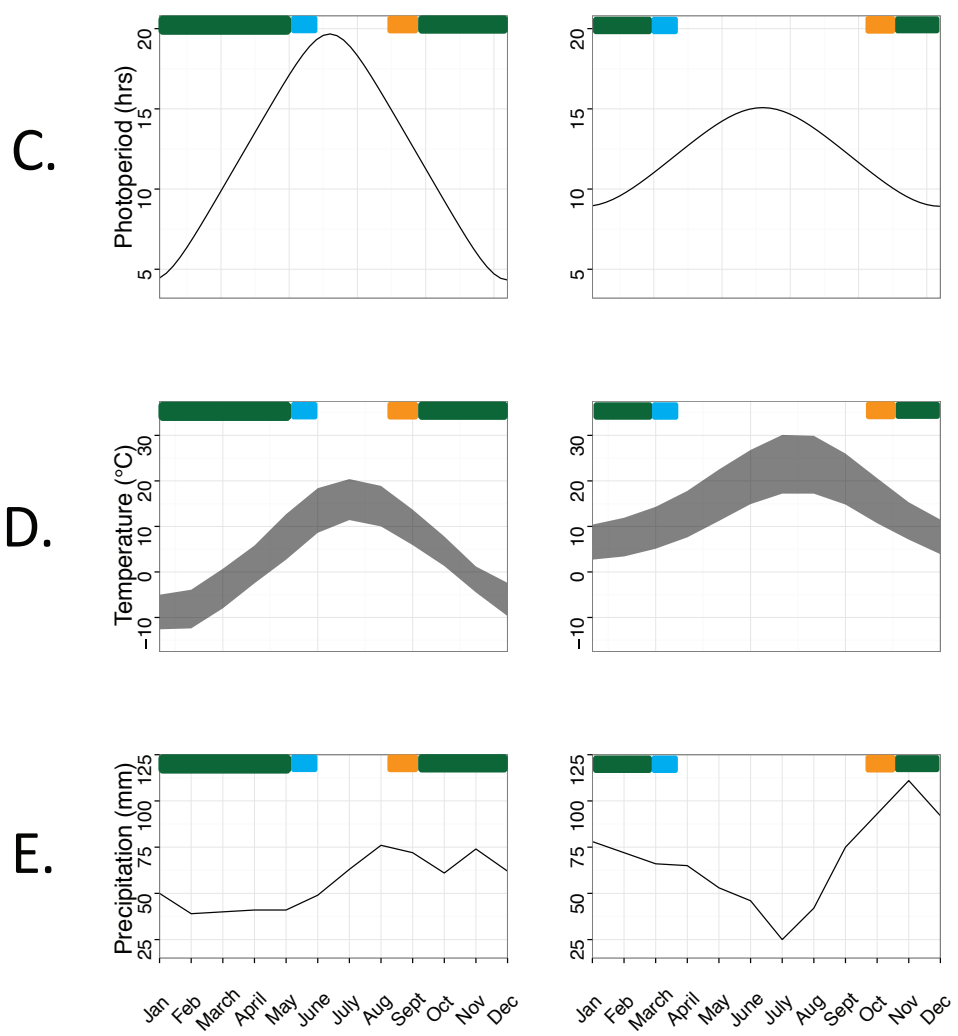

Figure 1. Water-use related environmental data for Sweden and Italy sites. Horizontal bars at the top of graphs indicate the growth stage intervals: germination period (orange), growing season (green), and flowering period (blue). A, Weekly Normalized Difference Vegetation Index (NDVI; mean \pm SD). B, Weekly frequencies of observed drought conditions of varying severity, based on Vegetative Health Index. C, Weekly length of photoperiod. D, Monthly maximum and minimum temperature. E, Monthly average precipitation. 


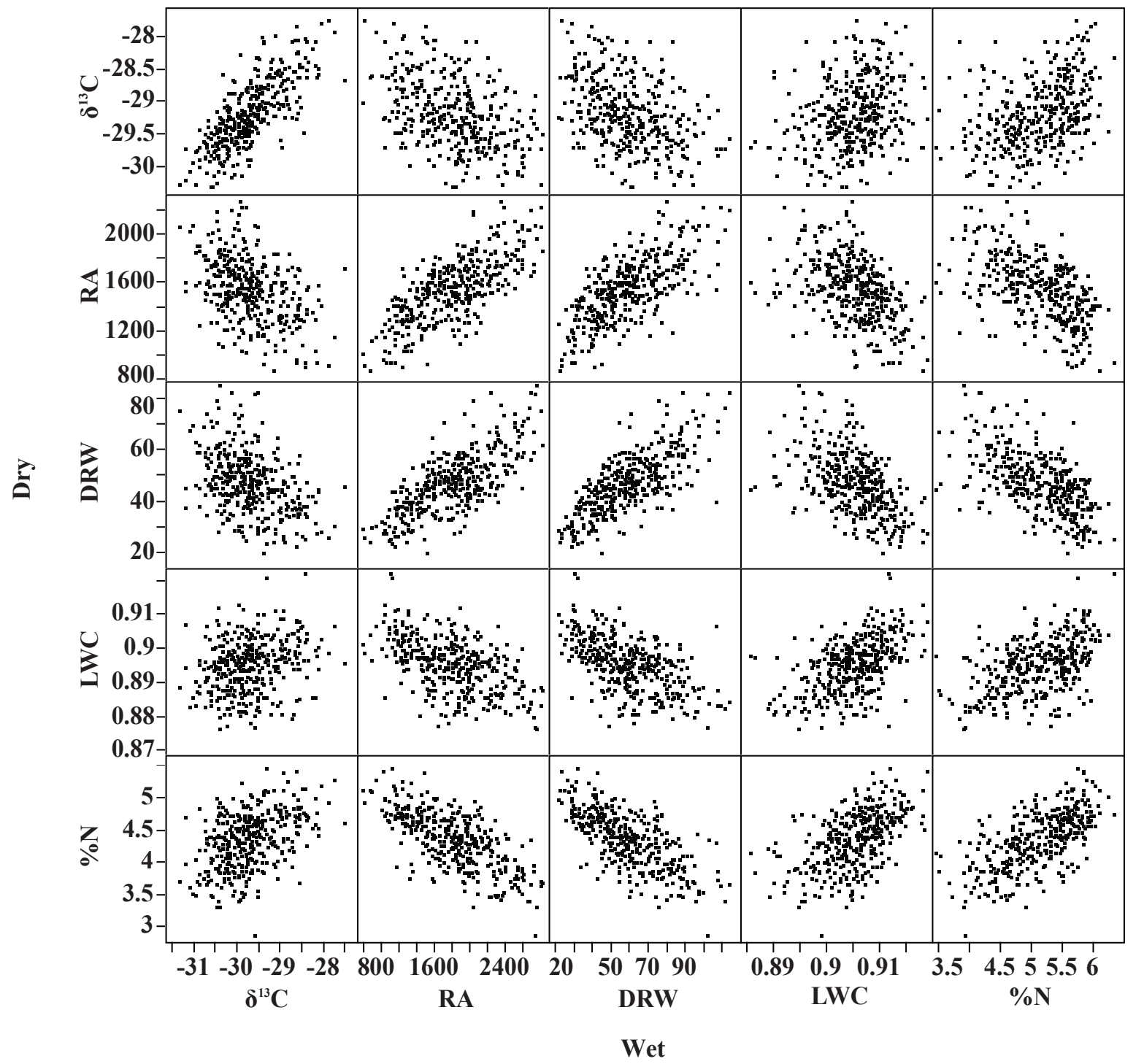

Figure 2. Genetic correlations of water-use physiology traits between wet and dry treatments. Data points represent least square mean estimates for individual recombinant inbred lines. All genetic correlations were highly significant (Pearson correlation, $\mathrm{P}<0.0001$ ). DRW, dry rosette weight (g); LWC, leaf water content $(\%)$; RA, rosette area $(\mathrm{mm} 2) ; \delta 13 \mathrm{C}$, carbon isotope discrimination $(\%) ; \% \mathrm{~N}$, leaf nitrogen (\%). 


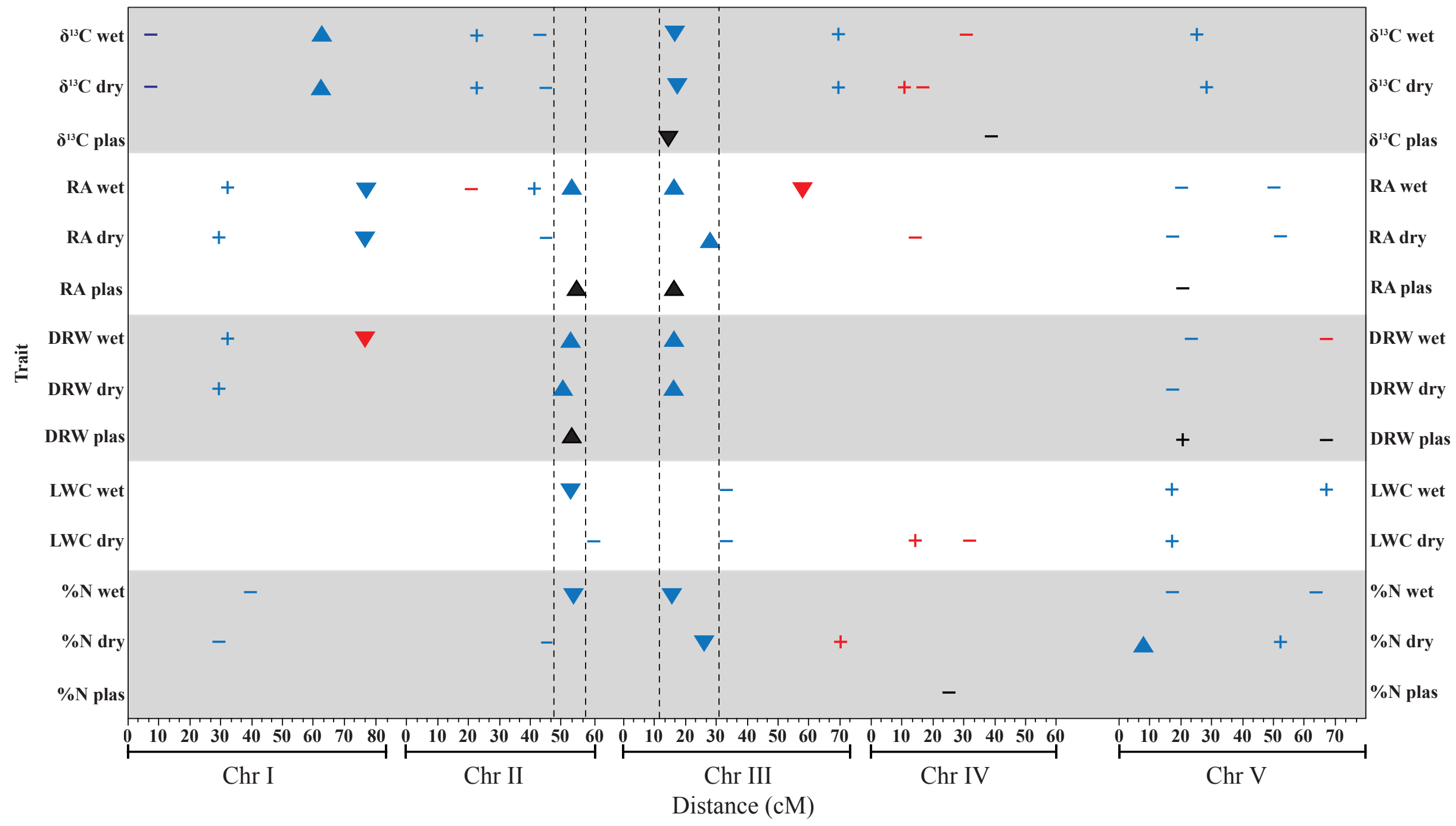

Figure 3. Location and direction of effect of water-use physiology QTL in the Italy $x$ Sweden mapping population. Plus sign indicates that the Swedish allele increased the trait value relative to the Italian allele; minus sign indicates the Swedish allele decreased the trait value. Upward triangle indicates that the Swedish allele increases the trait value and that the QTL co-localizes with a fitness QTL from Ågren et al. (2013); downward triangle indicates that the Swedish allele decreases the trait and that the QTL co-localizes with a fitness QTL. Blue symbols represent constitutive QTL, red symbols represent inducible QTL, and black symbols represent plasticity QTL. Vertical dashed lines indicate hotspot regions. DRW, dry rosette weight; LWC, leaf water content; RA, rosette area; $\delta 13 \mathrm{C}$, carbon isotope discrimination; \% $\mathrm{N}$, percent leaf nitrogen. 